\section{ADHD MONITORING: AUDIT OF NICE GUIDELINES}

Anna-Louise Power, Elham Abbas. United Kingdom

\subsection{6/bmjpo-2021-RCPCH.125}

Background ADHD is a complex, diverse and common neurodevelopmental disorder, associated with many comorbidities. NICE guidelines set out the monitoring requirements for children being treated for ADHD. Wigan has more than 1000 children on its database with ADHD on medication, mostly on stimulant medication. Monitoring of the effectiveness of medication and adverse effects is crucial. Adverse effects on growth, heart rate, blood pressure changes and worsening behaviour may significantly impact the child if left unmonitored.

Objectives To review children who are on ADHD medication and to ensure parameters are monitored as per the NICE \& Regional GM guidelines in order to improve our patient care. Methods 47 patients who attended clinic in November 2018 and completed a symptom rating scale at this clinic visit were reviewed. The notes for these patients were reviewed for every clinic visit for these patients in the period January 2018 - July 2019.

Results In total we reviewed 118 clinic visits in 47 patients. $28 \%$ were age under 10 and $72 \%$ over 10 . Of these $30 \%$ of under 10 were seen the minimum of 3 times a year, whereas for the over tens $44 \%$ were seen the minimum of twice a year.

Core ADHD symptoms were recorded in 30\%. The effectiveness of the medication was discussed in $56 \%$ and the side effects in $9 \%$. The ongoing need for medication was only discussed once.

Height and weight were well documented in 97\% of cases, yet only $11 \%$ recorded the growth centile.

21/118 (18\%) clinic visits showed a loss in weight compared with previous visit, $33 \%$ of those had a plan to address the problem.

Cardiovascular side effects monitoring was poor, with heart rate measured in $65 \%$ and blood pressure in $78 \%$. Heart rate was found to be greater than 120 in one case but no action was taken. Blood pressure was only plotted on the centile chart in 1 case.

Symptoms like tics were discussed in 7\%, seizures $0 \%$ and Sleep 59\%. Worsening behaviour was discussed in 70\% cases.

Conclusions

Discussion

NICE guidelines recommend that children age under 10 should be reviewed at least 3 times per year and over 10 should be seen twice a year. In our audit only $30 \%$ of under 10 s were seen three times. Monitoring of patients on medication for ADHD was not good. Height and weight should be checked in all children but this is poorly plotted on the correct growth chart. Blood pressure and heart rate are not always being checked.

Conclusion

The audit highlighted gaps in monitoring of our ADHD patients. Following this audit, a side effect questionnaire was introduced for use at all clinic visits. Rating scale questionnaires were also introduced to assess the effectiveness of the medication. Also we appointed a clinical pharmacist, who completed his prescribing course. Our ADHD specialist nurse also completed the prescribing course to help the paediatricians review medication as per NICE recommendation.

\section{ESTABLISHING A RISK STRATIFICATION SCORING SYSTEM FOR IVIG RESISTANCE IN KAWASAKI DISEASE IN HONG KONG}

Chi Tak Enoch So, Yan Wah Cheng, Man Ching Yam, Lai Ling Hui, Albert Martin Li. Hong Kong

\subsection{6/bmjpo-2021-RCPCH.126}

Background Kawasaki disease (KD) is an acute febrile multiorgan vasculitic syndrome primarily affecting infants and children with potentially devastating cardiovascular sequelae. The mainstay treatment for $\mathrm{KD}$ is intravenous immunoglobulin (IVIG).

Regrettably, IVIG resistance occurs in a sizeable portion of patients. International guidelines advocate primary intensification therapy with adjunctive steroids, in addition to IVIG, as initial treatment for this sub-group of KD.

In order to enable the aforementioned potential, however, there is a need to establish a validated methodology which segregates high and low risk IVIG-resistance patients in Hong Kong.

Objectives Our primary objective is to determine the applicability of the current international risk scores in predicting IVIG resistance in Hong Kong Chinese.

Our secondary objective is to design a risk stratification scoring system for use in our local population.

Methods A 5-year retrospective case control (IVIG resistance vs. responsive) study (2015-2019) of patients aged 0-18 years diagnosed with KD from two tertiary hospitals was performed. Patients in which IVIG was never administered, or administered at another hospital, were excluded. Resistance cases were defined as relapse or persistent fever with temperature $\geq$ $38.0^{\circ} \mathrm{C}$ at least 36 hours after the end of first IVIG infusion. The remaining subjects were defined as responsive and grouped as controls.

Applying established international risk scores (Kobayashi, Egami, Sano, Harada, Fu) in our sample generated sensitivity, specificity, positive predictive value (PPV) and negative predictive values (NPV) of each score for comparison. Modification of Kobayashi and Sano scores were necessary by substituting AST (aspartate transaminase) with ALT (alanine transaminase) due to lack of data in our population.

In composing a clinical risk scoring system, multi-variate logistic regression model was constructed using variables selected by univariate analysis. Demographic variables and variables with $\mathrm{p}<0.1$ in univariate analysis were included. Continuous variables were converted to dichotomous variable based on 25 th or 75 th centile value. Backward elimination based on likelihood ratio was performed. A $\mathrm{p}$ value of $>0.1$ was required for elimination.

Results This study included 210 patients, of which 39 patients $(18.6 \%)$ were IVIG resistant. The median age was 21 months old (IQR 10.8 - 40.3), with 61.0\% male patients. Among all included risk scores, modified Kobayashi score performed best with sensitivity (53.8\%), specificity (74.9\%), PPV (32.8\%) and NPV (87.7\%).

Our preliminary analysis offered an alternative 4-variable model, code named HK-KD score $v 1$, based on gender, neutrophil percentage, urea, and globulin. Using an aggregate score cutoff of $\geq 6$ in defining high-risk IVIG resistant patients, HK-KD score v1 yielded sensitivity (66.7\%), specificity (78.4\%), PPV (41.3\%), and NPV (91.2\%). Higher aggregate score of $H K-K D$ score $v 1$ is suggestive of a higher chance of IVIG resistance. 
Conclusions Modified Kobayashi score performed best among the established international risk scores for IVIG resistance with good specificity but fair sensitivity in our population. We have designed a preliminary novel clinical risk score for IVIG resistance, $H K-K D$ score $v 1$, with sufficient sensitivity and specificity for potential clinical use.

\section{A NOVEL TWO-TIERED DEVELOPMENTAL SCREENING PROGRAMME FOR SINGAPOREAN TODDLERS: A QUALITY IMPROVEMENT REPORT}

Clara Nwe Nwe Linn Oo, Yoke Hwee Chan, David Chee Chin Ng, Truls Ostbye, Pratibha Keshav Agarwal, Sita Padmini Yeleswarapu. Singapore

\subsection{6/bmjpo-2021-RCPCH.127}

Background Early identification of developmental delays with timely intervention, especially before the age of 3 years, can optimise child development. With the pre-existing model of developmental surveillance in Singapore however, children with suspected developmental delays first consult a developmental specialist at a median age of 44 months to undergo a detailed developmental assessment and diagnosis, and only then receive intervention.

Objectives Our Quality Improvement team agreed that improvements made to the pre-existing model of developmental surveillance would enable earlier identification of developmental delays thereby triggering early intervention. Hence, we aimed to increase the proportion of children seen in primary care who screen positive for potential developmental delays by $5 \%$ before the age of 3 years, with the use of a novel twotiered developmental screening programme in a primary care setting, without increasing the false positive rates.

Methods We describe the implementation and evaluation of a novel two-tiered developmental screening programme into the existing 9-month and 18-month screening schedule, with an additional screening at 30 months to replace the pre-existing 36-month screening of the National Child Health Surveillance Programme. The new programme utilizes some of the American Academy of Pediatrics's recommended screening tools: Parents' Evaluation of Developmental Status (PEDS), Parents' Evaluation of Developmental Status - Developmental Milestones (PEDS-DM) and The Ages \& Stages Questionnaires (ASQ) as part of our new two-tiered screening programme. We evaluated this programme using quality improvement methods by measuring the proportion of children referred to child development units after positive screening for developmental delays under the new programme, with a pre-post and with-without comparison. We also studied risk factors associated with positive screening in the programme.

Results The two-tiered screening was most effective at 18 months with the proportion referred improving from $3.5 \%$ to $7.1 \%$ over a 6 -month period. In addition, the referred proportion at 18 months was also significantly higher at the primary care center where the two-tiered screening was implemented as compared to other primary care centres in the same healthcare cluster who were utilizing the pre-existing screening (7.8\% compared to $3.2 \%)$. For those who received further assessment by developmental specialists after the two-tiered screening, $100 \%$ received a definitive diagnosis of developmental delays, similar to our baseline data. The risk factors identified for screening positive among Singaporean toddlers: male gender, low maternal education, preterm births and low income shed light on population subgroups which may require more attention and resources in future.

Conclusions Our quality improvement efforts have facilitated the integration of a novel two-tiered screening programme into the pre-existing screening schedule, with improved referral proportions without increasing the screening process's false positive rates. While we highlight challenges in implementation that need to be addressed, our findings support a potential nation-wide adoption of the two-tiered programme.

\section{BEHAVIORAL ANALYSIS OF DRUG NON-COMPLIANCE IN PEDIATRIC EPILEPSY}

Fatima Mazahir, Fatima Mazahir. United Arab Emirates

\subsection{6/bmjpo-2021-RCPCH.128}

Background Epilepsy ranks among the commonest chronic neurological diseases, with a global estimate of 5 million people diagnosed annually. It is broadly defined as having two or more unprovoked seizures linked to a spectrum of behavioral, psychiatric, and cognitive disorders resulting from birth injuries, endemic conditions, or traumatic brain injuries. Worldwide, up to 50 million people are affected, of whom 10.5 million are children below 15 years of age. Despite its high burden, up to $70 \%$ of epileptic patients could live seizure-free with accurate and timely diagnosis and appropriate long-term treatment with relatively cheap and effective anti-epileptic drugs (AEDs). The main obstacle in achieving this goal is the medication non-compliance more frequently reported in middle-income (44.4\%) and low-income countries (74.8\%). Adherence relates to using the prescribed treatment appropriately with regards to time, quantity, period, and manner.

Objectives We aim to understand the behaviors underlying non-compliance to AEDs in children from marginalized communities to formulate specific interventions to improve the treatment gap.

Methods The target population was children diagnosed with epilepsy under 15 years from lower- and middle-income communities (LMIC), and non-adherence to AEDs was identified as the target behavior. A literature review with keywords 'Pediatric Epilepsy,' 'LMIC,' 'Non-Compliance,' 'Medication Adherence,' and 'Behaviors' was conducted via Google Scholar and PubMed. Based on the abstract review, 22 articles were shortlisted for full article review, out of which 7 articles were finalized for analysis. The primary factors identified were fear, stigma, concerns about the medication's safety, and insufficient knowledge of the disease. These behaviors were analyzed in detail via the COM-B Model of Capability, Opportunity, and Motivation proposed by Miche et al. This analysis provides a framework to link targeted interventions to the specified behaviors and policy domains to the outlined interventions via the Behavior Change Wheel (BCW) Methodology.

Results A wide range of physical, psychological, and social factors underpinning the non-compliance to the AEDs were identified and mapped to the COM-B Model (table 1) of psychological or physical capabilities, social and physical opportunities, and automatic and reflective motivation. Forgetfulness was the primary issue at around $54.25 \%$, followed by disapproved perceptions about epilepsy such as denial, selfpity, social exclusion, and fear of societal exclusion $(20.55 \%)$; and mistrusting their physician leading to increased concerns about medication safety and side-effects (8.87\%). 